\title{
Beneficial effects of metformin on haloperidol-induced motor deficits in rats. A behavioral assessment
}

\author{
George Jîtcă'1,2, Zsolt Gáll1, Camil E Vari1, Bianca E Ősz¹, Amelia Tero-Vescan³, Alexandra Groșan¹, \\ Maria T. Dogaru 1 \\ 1. Department of Pharmacology and Clinical Pharmacy, Faculty of Pharmacy, George Emil Palade University of Medicine, Pharmacy, Science, and Technology \\ of Targu Mures, Romania \\ 2. Doctoral School of Medicine and Pharmacy, I.O.S.U.D., George Emil Palade University of Medicine, Pharmacy, Science, and Technology of Targu Mures, \\ Romania \\ 3. Department of Biochemistry, Faculty of Pharmacy, George Emil Palade University of Medicine, Pharmacy, Science, and Technology of Targu Mures, \\ Romania
}

\begin{abstract}
Objective: One of the most common side effects of haloperidol is the extrapyramidal syndrome, resulting from inhibition of nigrostriatal dopaminergic circuits and mitochondrial dysfunction due to structural similarities to pyridinium derivative, MPP+ that induce oxidative stress. In exchange, the use of metformin appears to enhance neurogenesis, energy metabolism, and oxidative status, so these properties can be speculated in the context of drug-induced pseudoparkinsonism by haloperidol. Methods: To assess motor coordination and activity, rodents were divided into four groups: CTR $(n=10)$ - animals that received distilled water, METF $(n=10)$ - animals that received metformin 500 mg / kgbw, HAL $(n=10)$ - animals that received haloperidol 2mg / kgbw, HALMETF ( $n=10)$ - haloperidol $2 \mathrm{mg} / \mathrm{kgbw}$ and metformin 500 mg / $\mathrm{kgbw}$. The treatment was administered for 34 days at the same time by gastric gavage, during which time behavioral tests, rotarod (days 7 , $14,21,28$ ), catalepsy (day 30), open field (day 32) and novel object recognition (day 34) were performed. Results: The monitored parameters, showed significant differences between the groups of interest (HAL and HALMETF respectively), so that the administration of metformin at the beginning of treatment reduces the cataleptic behavior. The HALMETF group shows an attenuation of the motor deficit during the rotarod test and the freezing period from the Open Field test, is diminished. Conclusions: Metformin treatment has a beneficial effect in haloperidol-treated rats, demonstrated by decreased cataleptic behavior, improved motor performance and reduced haloperidol-induced anxiety behavior.
\end{abstract}

Keywords: metformin, haloperidol, rat, behavior, coordination

Received 17 March 2021 / Accepted 28 May 2021

\section{Introduction}

Contrary to the fact that progreses have been made in the medical field, due to the complex physiology of the brain, for many of the mental disorders, pharmacological approaches could not meet the therapeutic needs [1]. In the case of schizophrenia but also in other neurotic disorders, despite the development of modern neuroleptics, one of the most widely used antipsychotics remains haloperidol given its low costs and high potency [2]. From a pharmacotherapeutic view, haloperidol exerts its effects on the central nervous system (CNS) through dopaminergic antagonistic action with competitive blocking of postsynaptic $\mathrm{D}_{2}$ dopaminergic receptors in the mesolimbic system [3]. Extrapyramidal effects, mainly pseudoparkinsonian symptoms are due to blockade of the same receptor subtype in the nigrostriatal dopaminergic circuit [4] and are often irreversible. In addition to this mechanism, the blockade of complex I (NADH : ubiquinone oxidoreductase) of the electron transport chain (ETC) could lead to the same extrapyramidal symptoms by the degeneration of dopaminergic neuronal mass. Structural similarity ex-

* Correspondence to: Zsolt Gáll

E-mail: zsolt.gall@umfst.ro ists between haloperidol and the pyridinium derivative of 1-methyl-4-phenyl-1, 2, 3, 6-tetrahydropyridine (MPTP) [5], 1-methyl-4-phenylpyridinium (MPP+) that causes mitochondrial dysfunction, and a considerable amount of evidence was already published that haloperidol induces oxidative stress $[6,7]$. Considering this structural similarity it was assumed that the ability of $\mathrm{HPP}^{+}$to penetrate the neuron is related to a specific transporter, hOCT3 $[8,9]$. Based on its property of interacting with neurotoxins and positively charged molecules, it was suggested that hOCT3 is an effective transporter of $\mathrm{HPP}^{+}$. Also, it contributes to the exacerbation of the irreversible extrapyramidal effects of this neurotoxic metabolite. Thus, dopamine depletion or blockade of dopaminergic receptors causes an imbalance of monoaminergic metabolism that further leads to defects, especially motor impairment.

Considering the fact that research on human subjects is restricted, most of the data on behavioral and neurochemical effects come from animal models. Many of these studies present data obtained after acute treatment, but the therapeutic efficacy occurs over time, which implies that a more appropriate animal model is that obtained after chronic treatment [10].

Drug-repurposing, a promising strategy to accelerate the drug discovery process, has already been suggested for met- 
formin $[11,12]$. In addition, rodent studies indicate that chronic use of metformin prevents the adverse effects of aging and increases life expectancy $[13,14]$, and data from a meta-analysis confirms decreased risk of Parkinson's disease in combination with sulfonylurea derivatives [15]. As it was previously demonstrated, the effects of metformin occur are attributed to the AMP-activated protein kinase (AMPK) activation and phosphorylation followed by increased peroxisome proliferator-activated receptor gamma coactivator 1-alpha (PGC-1 $\alpha$ ) expression $[1,16]$ of muscle coordination and activity [17]. In vitro models show that metformin is able to stop the apoptotic process via AMPK pathway [18], being a possible therapeutic target in neurodegenerative diseases [7]. However, the effects of metformin on the CNS of experimental animals are poorly studied. Even if metformin is able to block the complex I of the electron transport chain, the doses of metformin used do not produce reactive species $[1,19]$.

Therefore, the aim of this study, was to investigate the influence of metformin on the behavioral changes induced by haloperidol, and its ability to prevent or delay the onset of neuroleptic-induced parkinsonian symptoms in rats.

\section{Material and Methods}

The study was conducted in compliance with all experimental procedures in accordance with European Directive 2010/63 / EU and was approved by the Ethics Committee for Scientific Research of the George Emil Palade University of Medicine, Pharmacy, Science and Technology of Târgu Mureș (approval no. 533/2019), and by National Sanitary Veterinary and Food Safety Authority (approval no. 42/2020).

\section{Animals and Treatment}

Haloperidol (Haloperidol Richter 2 mg / ml, Gedeon Richter, Târgu Mureș, Romania) and metformin (Glucophage 500 mg, Merck Santé, Semoy, France) were purchased from the Romanian pharmaceutical market. The protective coating of the tablets was removed and tablets were powdered. The powder was then added to distilled water which served as a solvent, resulting an extemporae suspension. The experiment was performed on a number of 40 male Wistar rats aged 6 months and an average weight $( \pm$ SEM) of $453.3 \pm 2.1$ grams, from the Animal Facility of the George Emil Palade University of Medicine, Pharmacy, Science and Technology of Târgu Mureș. Upon arrival in the laboratory, the animals were placed in individual cages, with a habituation period of 7 days, handled daily for stress reduction, while the environmental conditions were standard (12/ 12 hours light-dark cycle), ambient temperature $20 \pm 2^{\circ} \mathrm{C}, 60 \% \pm 10 \%$ humidity. The access to food, which consists of standard pellets for rodent, was unaltered throughout the study. Body weight was recorded once a week for dose adjustment.

Rodents were randomly divided into 4 groups: CTR (Control, distilled water, $\mathrm{n}=10$ ), HAL (haloperidol $2 \mathrm{mg}$ / kg, $\mathrm{n}=10$ ), METF (metformin $500 \mathrm{mg} / \mathrm{kg}, \mathrm{n}=10$ ), HALMETF (haloperidol $2 \mathrm{mg} / \mathrm{kg}$ and metformin 500 $\mathrm{mg} / \mathrm{kg}, \mathrm{n}=10$ ). The medication was administered for a period of 34 days, daily, between 8:00 AM and 10:00 AM with the help of an oral feeding cannula, in a volume of $1 \mathrm{ml} / \mathrm{kg}$, in a separate room. Administration dosages, administration route and treatment duration were established based on literature findings [20, 21, 22]. Also, the sample size per each group was chosen by consulting other similar experiments on rats from the literature [23, 24].

At the end of the experiment, all animals were decapitated under anesthesia with ketamine / xylazine in a dose mixture of ketamine $(100 \mathrm{mg} / \mathrm{kg})$ and xylazine $(10 \mathrm{mg}$ $/ \mathrm{kg}$ ), in order to collect samples of neuronal tissue and blood for further investigations.

\section{Behavioral assessment}

The behavioral tests were performed 6 hours after the administration of the medication (except for the catalepsy test), between 14: 00-20: 00, in order to reduce the possible confounding effects of sedation and catalepsy manifested immediately after the administration of haloperidol Study timeline of the chronic experiment and the timing of behavioral assays is shown in Figure 1. The investigators were not blinded to animal group during experiments, but the offiline behavioral analysis was performed by two experienced researchers to asses inter-observer reliability and to reduce bias. During the experiment we did not consider the measurement of glycaemic levels because the stress induced in order to determine this parameter might affect experimental animal behavior and based on the fact that metformin does not influence glycaemic levels this deter-

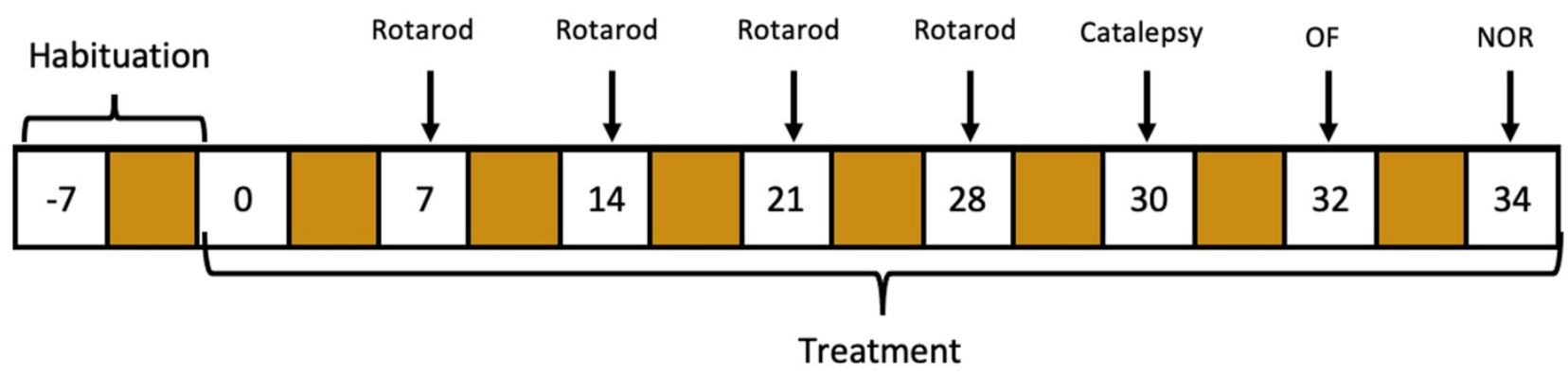

Fig. 1 Study timeline of chronic experiment and the timing of behavioral assays. The timeline shows the treatment period of 34 days preceded by a regular period of 7 days (OF - Open Field, NOR - Novel Object Recognition). Behavioral tests were performed between 14:00 and 20:00 PM. 
mination was not deemed justified. Moreover the authors did not consider this parameter to be involved in the protective effect.

\section{Catalepsy}

The cataleptic effect in rats was assessed by performing the bar test method on day 30 of the experiment. The behavior of the rats was evaluated 30 minutes after medication and then at one-hour intervals, up to 180 minutes. The height of the bar was set at $10 \mathrm{~cm}$ and the rats were carefully positioned, with the front limbs on the bar and the hind limbs on the floor of the apparatus. The end point of the catalepsy test was considered to be the moment when both forelegs were removed from the bar or the situation in which the animal moved its head, noting the desire to explore. If the rodents failed to maintain on the bar from 3 attempts, it was considered that there is no catalepsy phenomenon. The cut-off time was considered 300 seconds. After each trial the animals were placed back in the individual cage [25].

\section{Locomotor performance and coordination}

The motor coordination was assessed on a rotarod apparatus (Ugo Basile 7700, Italy) and this test is one of the most widely used for assessing the motor coordination ability of rats. The speed was constant during the analysis, in order to obtain a higher sensitivity [26], and the endpoint of this test was considered as the moment when the animal falls from the device. The device is attached to a drum with a diameter of $5 \mathrm{~cm}$ that rotates depending on the speed applied by the motor. The apparatus is divided into 4 equal compartments by separating plates.

Subjects were placed in the experimental room at least one hour before the experiment in order to become accustomed to the environment. For training, the rats were placed on the rotarod at a speed of $15 \mathrm{rpm}$ for 180 seconds [8], 5 consecutive days, before starting the treatment, in order to reach a stable performance. During treatment, rodents were tested weekly at a speed of $15 \mathrm{rpm}$ and latency was measured until falling or for maximum of 300 seconds. After each training session and test, the device was cleaned with $70 \%$ alcohol; the temperature at which the workouts and tests were performed (once a week for 4 weeks) was maintained at $20 \pm 2{ }^{\circ} \mathrm{C}$ in a quiet room.

\section{Open Field}

The Open Field (OF) test was performed in a plexiglass box of $60 \times 60 \mathrm{~cm}$ with black base and transparent walls, with a height of $50 \mathrm{~cm}$. During the test, the animals were placed in the center of the arena. The motor and behavioral activity were recorded using a top view camera at $30 \mathrm{fps}$, for 5 minutes [27]. The arena was disinfected after each test with $70 \%$ alcohol. All trials were analyzed with EthoVision XT (Noldus IT, Wageningen, The Netherlands, version 11.5), by monitoring the moving distance of the rats and their immobilization time.

\section{Novel object recognition}

Novel object recognition test (NOR) was used to evaluate memory. The objects used were about the same height and were heavy enough so that the rodents were unable to move them from their original position. They were placed at an equal distance of $30 \mathrm{~cm}$ from the corners, diametrically opposite and after each analysis were wiped with $70 \%$ alcohol to limit the appearance of any olfactory bias.

Initially, two identical objects were placed for each rat for a period of 5 minutes. At the end of this period, they were returned to the personal cage, at which point the box and objects were cleaned. Subsequently, after 24 hours, the procedure was repeated, but one of the familiar objects was replaced with a new one in order to evaluate the retention process. This test lasted for 5 minutes. Exploratory activity was considered valid when rodents sniff objects or touch them with their front paws but without leaning on or sitting on them. The count of the exploration times was performed with the help of the recording software. In this context, the discrimination index (DI) represents the difference of exploration time of the new object (EB) compared to the familiar object (EA) compared to the total time spent exploring the two objects in the retention process, $\mathrm{DI}=(\mathrm{EB}-\mathrm{EA}) /(\mathrm{EA}+\mathrm{EB})[28,29,30]$.

\section{Statistical analysis}

Statistical analysis was performed using Graphpad Prism software (San Diego, California USA, ver. 8). The Kolmogorov-Smirnov test was performed to test the normality of the data and values were expressed as mean \pm SEM. For the statistical analysis of catalepsy and rotarod test results, a two-way ANOVA followed by Tukey's post-hoc test was performed to evaluate the influence of the treatment. For statistical analysis of the OF and NOR tests, one-way ANOVA test was performed. The significance level was set at $p$ value less than 0.05 .

\section{Results}

\section{Catalepsy}

All groups were subjected to the same test, but the CTR and METF groups did not show cataleptic states. There was a significant effect of the treatment $\left(\mathrm{F}_{3,144}=302.7\right.$, $\mathrm{p}<0.0001)$, time $\left(\mathrm{F}_{3,144}=43.01, \mathrm{p}<0.0001\right)$ and a significant treatment $x$ time interaction $\left(\mathrm{F}_{9,144=} 13.91, \mathrm{p}<0.0001\right)$. Regarding the latency differences between HAL and HALMETF, significant differences were observed regardless the time of testing. Figure $2 \mathrm{~A}$ presents the latency to step down in the catalepsy test for rats of the METF, HAL, HALMETF and CTR groups, respectively. An overall increase in the latency to step-down from the bar at 30,60, 120, and 180 min after the administration was observed in HAL and HALMETF groups in relation to the CTR group.

HAL group showed an increasing tendency with time, a higher degree of catalepsy being observed at 120 and 180 minutes after administration (Figure 2B). On the other 


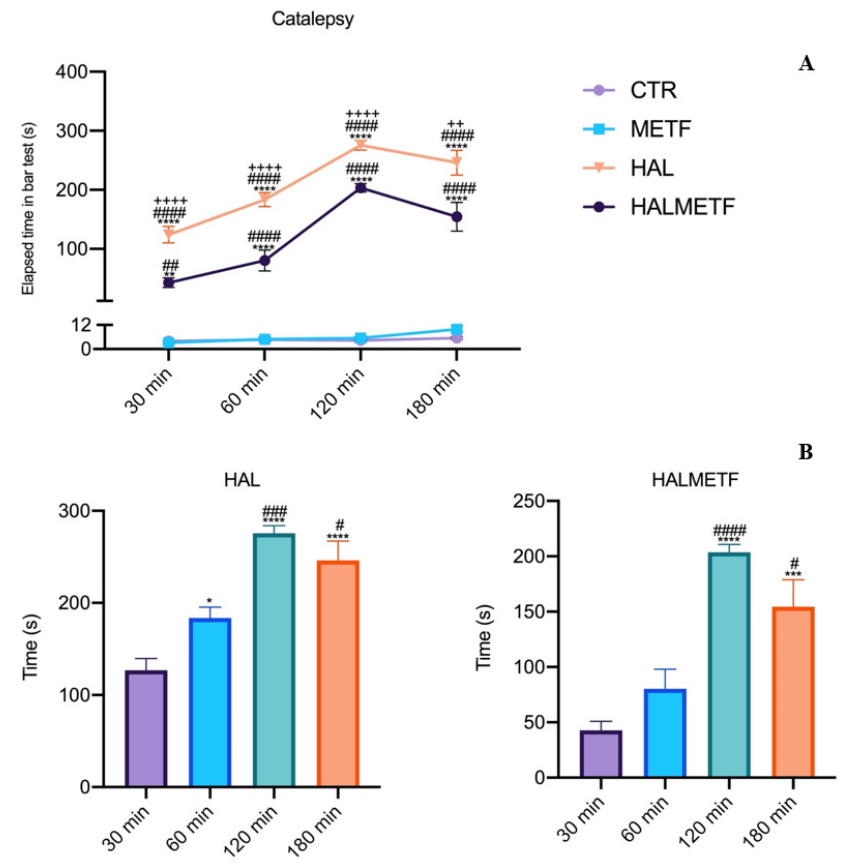

Fig. 2. (A) Latency to step down in the catalepsy test for rats expressed as seconds. Values displayed are means \pm SEM. Statistically significant differences compared to the CTR group (Control group) are noted with ${ }^{* \star} p<0.01,{ }^{* * \star *} p<0.0001$. Statistically significant differences compared to the METF group (Metformin group) are noted with $\# \# p<0.01$, \#\#\#\#p<0.001, Statistically significant differences compared to the HALMETF group (Haloperidol + Metformin group) are noted with $++p<0.01,++++p<0.0001$.

(B) Differences of latency to step down in the catalepsy test for group HAL (Haloperidol group) and HALMETF. Results are expressed as mean \pm SEM. Significant differences between treatment intervals are noted with ${ }^{*} \mathrm{p}<0.05,{ }^{* \star *} \mathrm{p}<0.001{ }^{* * * *} \mathrm{p}<0.0001$, compared to $30 \mathrm{~min}$. Significant differences between treatment intervals are noted with $\# p<0.05, \# \# \# p<0.001$, \#\#\#\#p<0.0001 compared to $60 \mathrm{~min}$.

hand, there is a similar trend of decrease of the latency to step down of the bar in the case of rats from the HALMETF group (Figure 2B), the time of maintaining the cataleptic state being shorter than in the HAL group.

\section{Rotarod}

To determine the effect of metformin on the ability to move, we tested the motor performance using the rotarod test. Rats were tested weekly by quantifying the time spent on the rod. The performance of the animals expressed as latency to fall off the rotating drum is shown in Figure 3A. A progressive decline in the case of HAL and HALMETF groups was observed. Two-way repeated measures ANOVA indicated a significant effect of treatment $\left(\mathrm{F}_{3}\right.$, $\left.{ }_{144}=505.8, \mathrm{p}<0.0001\right)$, time $\left(\mathrm{F}_{3,144}=33.23, \mathrm{p}<0.0001\right)$ and a significant treatment $x$ time interaction $\left(\mathrm{F}_{9,144}=23.22\right.$, $\mathrm{p}<0.0001)$. The Tukey post hoc test showed an overall decrease in the performance and motor coordination from the first to the fourth week of treatment (Figure 3A).

Within the HAL group, a sudden decrease in motor performance was observed in the second week, maintaining this trend in weeks 3 and 4 . At the same time, differences were found between week 2 - week 3 and week 2 - week 4, respectively (Figure 3B). In the HALMETF group a

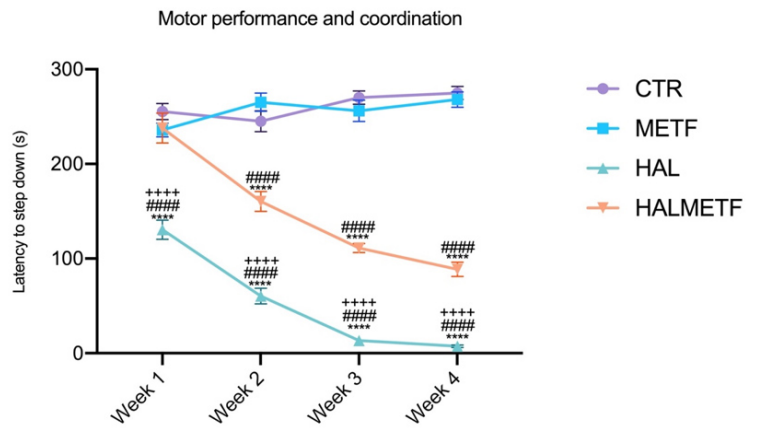

A
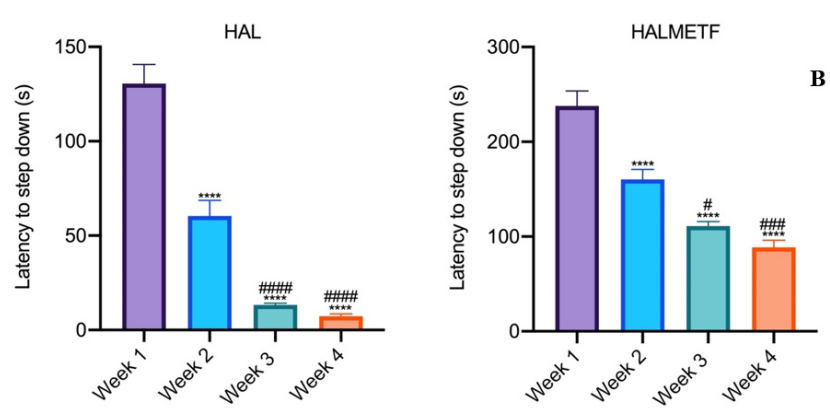

Fig. 3. (A) Motor performance and coordination using rotarod test. Values are expressed as mean \pm SEM. Statistically significant differences compared to the CTR group (Control group) are noted with ${ }^{* * * *} p<0.0001$. Statistically significant differences compared to the METF group (Metformin group) is noted with $\# \# \# \#<<0.0001$. Statistically significant differences compared to the HALMETF (Haloperidol + Metformin group) group is noted with $++++p<0.0001$. (B) Differences of latency to fall in the rotarod test for group HAL (Haloperidol group) and HALMETF. Results are expressed as mean \pm SEM. ${ }^{* * *} p<0.0001$, different from the week $1 ; \# p<0.01, \# \# \# p<0.001, \# \# \# p<0.0001$, different from week 2.

slight decrease in motor performance and coordination was observed on week 2 of chronic treatment administration. Moreover, the difference between week 3 and 4 the difference was not significant, suggesting a protective effect of treatment on motor capacity.

\section{Open field}

In order to establish the influence of medication (HAL, METF, HALMETF) on motor activity and immobilization time, the $\mathrm{OF}$ test was performed. A decrease in locomotor activity in the HAL and HALMETF groups was noted (Figure 4A). The freezing time in OF also demonstrated that the HAL group was the most affected (Figure $4 \mathrm{~B})$. However, the HALMETF group showed higher values of distance traveled and decreased time of immobilization.

\section{Novel object recognition}

Following the statistical analysis, it was not possible to determine exactly whether the combination with metformin improves the ability to discriminate between objects, so other parameters were also taken into account, such as distance traveled. Following the one-way ANOVA for the determination of DI (Figure 5A), no significant difference was found between the groups included in the study $\left(\mathrm{F}_{3}\right.$, $\left.{ }_{26}=0.1849, \mathrm{p}=0.9057\right)$. A negative DI value was found in the HAL group. In order to calculate the DI, only those 

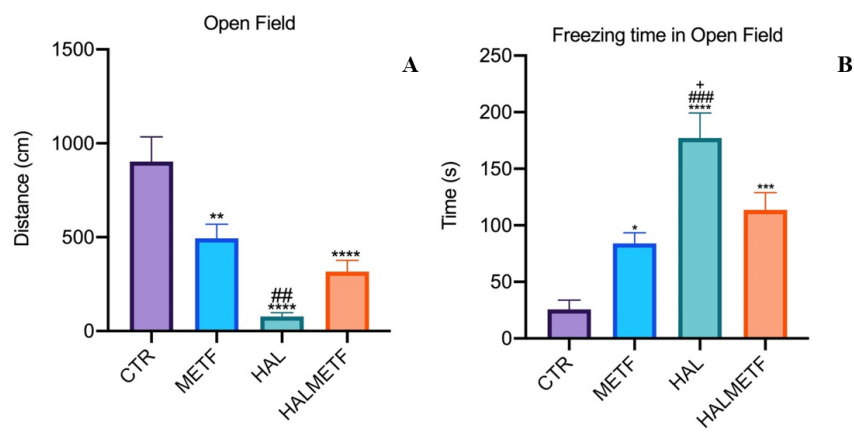

Fig. 4 Locomotor activity and freezing time in the Open Field test. Values are expressed as mean \pm SEM. Statistically significant differences compared to the CTR group (Control group) are noted with ${ }^{*} p<0.05,{ }^{* *} p<0.01,{ }^{* \star *} p<0.001,{ }^{* * \star *} p<0.0001$. Statistically significant differences compared to the METF (Metformin group) group

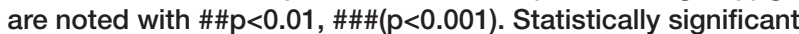
differences compared to the HALMETF group (Haloperidol + Metformin group) are noted with $+p<0.05$; $\mathrm{HAL}-$ Haloperidol group.

animals that showed a cumulative time spent with the two objects of at least one second were considered for analysis $($ CTR $\mathrm{n}=10, \operatorname{METF} \mathrm{n}=7, \operatorname{HAL} \mathrm{n}=5, \operatorname{HALMETF} \mathrm{n}=$ 8). Moreover, the freezing time was also evaluated, which reflected the existence of a state of anxiety, manifested in terms of neophobia, as a result of placing the two objects in the test environment. Significant differences were reported between the HAL group compared to the other groups, $\left(\mathrm{F}_{3,36}=9.435, \mathrm{p}<0.0001\right)$, as can be seen in Figure 5B.

To evaluate the motor activity in the Retention trial, the one-way ANOVA test revealed the existence of significant differences between the HAL group and CTR and METF

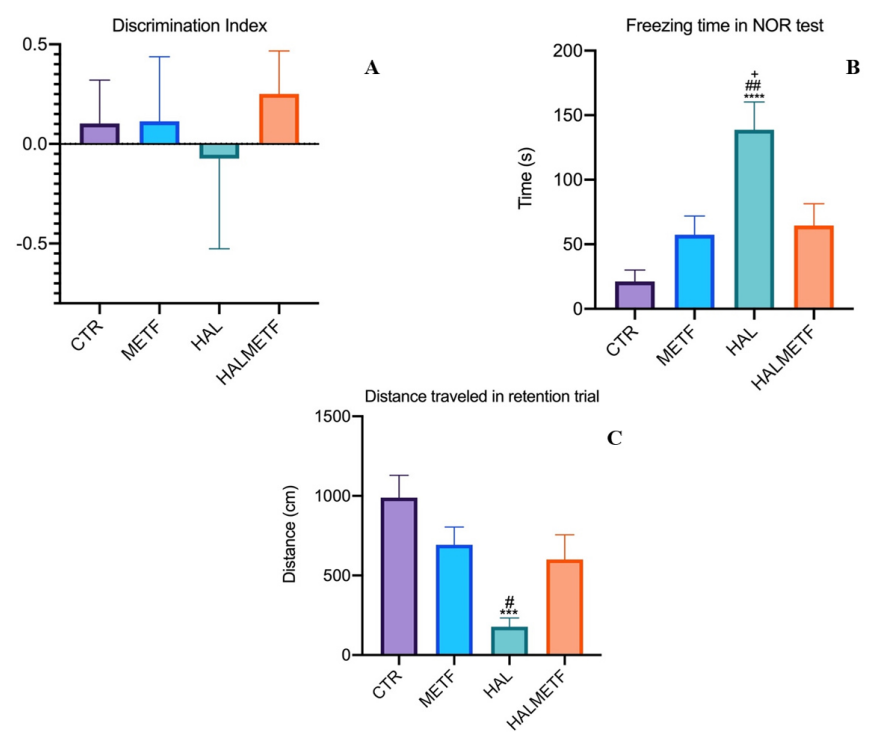

Fig. 5. (A) The effect of chronic treatment with haloperidol and metformin on the discrimination index (DI) of the familiar object and the new object in the retention process, (B) on the period of immobilization during the retention process and $(C)$ on the travel distance in the exploration stage. Data are expressed as mean \pm SEM, ${ }^{* * *} p<0.001,{ }^{* * * *} p<0.0001$ compared to CTR group, $\# p<0.05$, \#\#p $<0.01$ compared to METF group, $+p<0.05$ compared to HALMETF group. Note that the DI represents the average only of those animals that showed the cumulative curiosity for the two objects, for at least one second (CTR $n=10, \operatorname{METF} n=7$, HAL $n=$ 5, HALMETF $n=8$ ); HAL - Haloperidol group, HALMETF - Haloperidol + Metformin group. groups, respectively $\left(\mathrm{F}_{3,35}=7.584, \mathrm{p}=0.0005\right)$, as can be seen in Figure 5C.

\section{Discussion}

It is generally accepted that haloperidol, a non-selective dopaminergic receptor antagonist, possess the property of providing an empiric experimental model of Parkinson's disease in rodents due to its anti-dopaminergic mechanism of action and neurotoxicity, favoring bradykinesia and late dyskinesia [31]. However, the most obvious manifestation produced by haloperidol is catalepsy, following $\mathrm{D}_{2}$ receptor blockade $[16,32]$.

The results of this study demonstrate an improvement in cataleptic behavior and motor performance induced by haloperidol, following chronic treatment with metformin. Thus, in the catalepsy test, metformin significantly reduced the latency of descent from the bar in all tests performed, regardless of the time. These results are in agreement to those previously presented by Adedeji et al. [33], that metformin reduces the period of immobilization and improves the ability of behavioral adaptation [34].

The measurement of motor performance, coordination and balance in the case of the rotarod test, demonstrates a clear deficit in the case of the HAL group, with low latencies of falling off the drum rotating at a constant speed. Another detail worth mentioning is that these small latencies observed after the first week of treatment, are worsening throughout continuation. Interestingly, in the case of metformin, this latency has a less steep slope over time. Indeed, weight and weight gain may have a negative impact on rotarod performance, but rodents were pre-tested and weighed during the accommodation period and body mass remained constant throughout treatment. Therefore, it is unlikely that their performance was affected by weight. Motor dysfunction is obviously associated with the loss of dopaminergic neurons in the nigrostriatal level and dopamine depletion, because these neurons show projections to the striatum and their destruction leads to changes in the neural circuits in the basal ganglia, which are essential in regulating various movements. Thus, the association with metformin shows a significant improvement in motor activity, being consistent with other studies performed on small rodents [35].

In the OF test, there were no significant differences between the groups. However, compared to the HAL group, those who received the drug combination traveled longer distances.

Using the NOR paradigm we tried to assess the cognitive deficit induced by chronic haloperidol treatment and the ability of metformin treatment of preventing it. In addition, a reduction in immobilization time was noted in the presence of the two objects placed in the test environment.

Moreover, based on the influence exerted on the energy metabolism, evidence suggests that the therapeutic potential of metformin comes from the property of regulating the mitochondrial function, which could also affect be 
influenced at the central nervous system level (metformin can cross the blood-brain barrier) [13]. Thus, the clinical use of this compound remains to be investigated, and whether it could counteract the neurotoxic effects of haloperidol, such as mitochondrial impairments or disorders of energy metabolism in the brain.

This study confirmed that metformin can improve some of the behavioral alterations induced by haloperidol. Significant attenuation of cataleptic status, improved motor coordination, and attenuation of anxiety-like behavior have been observed. The METF group indicates a shorter immobilization period, in both OF and NOR tests, which could suggest that metformin decreases the anxiety-like behavior, but this hypothesis needs further consideration in future studies [36]. The evaluation of other cognitive functions, such as reversal learning and associative memory, brings additional information in the case of the development of motor and cognitive deficits. The clinical utility of metformin and its influence on energy metabolism and central nervous system function should be investigated in future studies to establish effects in neurological disorders. From the authors perspective, the increase in dosage regimen or in treatment duration would not necessarily lead to an improvement of the analyzed parameters. It is likely that with the increase, the blockage of the complex I of ETC by metformin would occur in a higher measure and the risk of developing lactic acidosis would also increase. Several studies emphasize the reduction of life expectancy on experimental animals $[14,37]$.

The present study comes with several limitations. First, the animals were individually placed and kept during the experiment, which presumes social isolation inducedstress. Second limitation is related to the fact that the tests were performed during day-time, although it is known that rats are nocturnal animals and their activity is intensified during night-time.

\section{Conclusion}

Metformin treatment may have a beneficial effect in haloperidol-induced motor dysfunction in rats, demonstrated by decreased cataleptic behavior and improved rotarod performance. Furthermore, an anxiolytic effect of metformin could also be indicated by a decreased freezing time and increased exploratory behavior, but further studies will be needed for confirmation.

\section{Author's contribution}

GJ - Data collection, conceptualization, investigation, writing the original draft, funding acquisition

ZG - Data collection, writing, editing, supervision

CEV - Writing and Editing

BEŐ - Writing and Editing

AMT - Writing and Editing

AG - Writing and Editing

MTD - Writing and Editing, conceptualization, supervision

\section{Aknowledgements}

This work was supported by the George Emil Palade University of Medicine, Pharmacy, Science, and Technology of Târgu Mureș, Research Grant number 293/4/14.01.2020.

\section{Conflicts of Interest}

The authors declare no financial or other conflict of interest.

\section{References}

1. Sportelli C, Urso D, Jenner P, Chaudhuri KR. Metformin as a Potential Neuroprotective Agent in Prodromal Parkinson's Disease-Viewpoint. Front. Neurol. 2020, 11: 556.

2. Sykes DA, Moore $H$, Stott $L$ et al. Extrapyramidal Side Effects of Antipsychotics Are Linked to Their Association Kinetics at Dopamine D2 Receptors. Nat. Commun. 2017, 8 (1): 763.

3. Mori T, Ito S, Kita T. Sawaguchi T. Effects of Dopamine- and SerotoninRelated Compounds on Methamphetamine-Induced Self-Injurious Behavior in Mice. J. Pharmacol. Sci. 2004, 96

4. Wiley, J. L. Antipsychotic-Induced Suppression of Locomotion in Juvenile, Adolescent and Adult Rats. Eur. J. Pharmacol. 2008, 578 (2-3), 216-221.

5. Balijepalli S, Kenchappa RS, Boyd MR, Ravindranath V. Protein Thiol Oxidation by Haloperidol Results in Inhibition of Mitochondrial Complex I in Brain Regions: Comparison with Atypical Antipsychotics. Neurochem. Int. 2001, 38 (5), 425-435.

6. Reinke A, Martins MR, Lima MS, Moreira JC, Dal-Pizzol F, Quevedo J. Haloperidol and Clozapine, but Not Olanzapine, Induces Oxidative Stress in Rat Brain. Neurosci. Lett. 2004, 372 (1-2), 157-160.

7. Lu M, Su C, Qiao C, Bian Y, Ding J, Hu G. Metformin Prevents Dopaminergic Neuron Death in MPTP/P-Induced Mouse Model of Parkinson's Disease via Autophagy and Mitochondrial ROS Clearance. Int. J. Neuropsychopharmacol. 2016, 19 (9), pyw047.

8. Kang $\mathrm{HJ}$, Lee SS, Lee $\mathrm{CH}$ et al. Neurotoxic pyridinium metabolites of haloperidol are substrates of human organic cation transporters. Drug Metab. Dispos. 2006, 34 (7), 1145-1151.

9. Wu X, Kekuda R, Huang $W$ et al. Identity of the Organic Cation Transporter OCT3 as the Extraneuronal Monoamine Transporter (Uptake2) and Evidence for the Expression of the Transporter in the Brain. J. Biol. Chem. 1998, 273 (49), 32776-32786.

10. Schmitt U, Dahmen N, Fischer $V$ et al Chronic Oral Haloperidol and Clozapine in Rats: A Behavioral Evaluation. Neuropsychobiology 1999, 39 (2), 86-91.

11. Pryor, R.; Cabreiro, F. Repurposing Metformin: An Old Drug with New Tricks in Its Binding Pockets. Biochem. J. 2015, 471 (3), 307-322.

12. Rena G, Lang CC. Repurposing Metformin for Cardiovascular Disease. Circulation 2018, 137 (5), 422-424.

13. Zuliani I, Urbinati C, Valenti D et al. The Anti-Diabetic Drug Metformin Rescues Aberrant Mitochondrial Activity and Restrains Oxidative Stress in a Female Mouse Model of Rett Syndrome. J. Clin. Med. 2020, 9 (6), 1669.

14. Martin-Montalvo A, Mercken EM, Mitchell SJ et al. Metformin Improves Healthspan and Lifespan in Mice. Nat. Commun. 2013, 4 (1), 2192.

15. Wahlqvist ML, Lee MS, Hsu CC, Chuang SY, Lee JT, Tsai HN. MetforminInclusive Sulfonylurea Therapy Reduces the Risk of Parkinson's Disease Occurring with Type 2 Diabetes in a Taiwanese Population Cohort. Parkinsonism Relat. Disord. 2012, 18 (6), 753-758.

16. Engelhard KA, Marchetta P, Schwarting RKW, Melo-Thomas L. Haloperidol-Induced Catalepsy is Ameliorated by Deep Brain Stimulation of the Inferior Colliculus. Sci. Rep. 2018, 8 (1), 2216.

17. Lu M, Chen H, Nie F, Wei X, Tao Z, Ma J. The Potential Role of Metformin in the Treatment of Parkinson's Disease. J. Bio-X Res. 2020, 3 (1), 2735.

18. Patil SP, Jain PD, Ghumatkar PJ, Tambe R, Sathaye S. Neuroprotective Effect of Metformin in MPTP-Induced Parkinson's Disease in Mice. Neuroscience 2014, 277, 747-754.

19. Rotermund C, Machetanz G, Fitzgerald JC. The Therapeutic Potential of Metformin in Neurodegenerative Diseases. Front. Endocrinol. 2018, 9, 400.

20. Pillai A, Dhandapani KM, Pillai BA, Terry AV Jr, Mahadik SP. Erythropoietin prevents haloperidol treatment-induced neuronal apoptosis through regulation of BDNF. Neuropsychopharmacology. 2008, 33 (8), 1942-51.

21. Zhang XF, Tan BK. Antihyperglycaemic and anti-oxidant properties of 
Andrographis paniculata in normal and diabetic rats. Clin Exp Pharmacol Physiol. 2000, 27(5-6), 358-63.

22. Tsai CM, Kuo HC, Hsu CN, Huang LT, Tain YL. Metformin reduces asymmetric dimethylarginine and prevents hypertension in spontaneously hypertensive rats. Transl Res. 2014, 164(6), 452-9.

23. Keshavarzi S, Kermanshahi S, Karami L, Motaghinejad M, Motevalian M, Sadr S. Protective role of metformin against methamphetamine induced anxiety, depression, cognition impairment and neurodegeneration in rat: The role of CREB/BDNF and Akt/GSK3 signaling pathways. Neurotoxicology. 2019, 72, 74-84.

24. Alharbi I, Alharbi $H$, Almogbel $Y$, Alalwan A, Alhowail A. Effect of Metformin on Doxorubicin-Induced Memory Dysfunction. Brain Sci. 2020, 10(3), 152.

25. Bricker B, Sampson D, Ablordeppey SY. Evaluation of the potential of antipsychotic agents to induce catalepsy in rats: assessment of a new, commercially available, semi-automated instrument. Pharmacol Biochem Behav. 2014, 120, 109-16.

26. Alhowail AH, Chigurupati S, Sajid S, Mani V. Ameliorative Effect of Metformin on Cyclophosphamide-Induced Memory Impairment in Mice. Eur Rev Med Pharmacol Sci. 2019, 23(21), 9660-9666.

27. Feyissa DD, Aher YD, Engidawork E, Höger H, Lubec G, Korz V. Individual Differences in Male Rats in a Behavioral Test Battery: A Multivariate Statistical Approach. Front Behav Neurosci. 2017, 11, 26.

28. Rajagopal L, Massey BW, Huang M, Oyamada Y, Meltzer HY. The novel object recognition test in rodents in relation to cognitive impairment in schizophrenia. Curr Pharm Des. 2014, 20(31), 5104-14.

29. Antunes M, Biala G. The novel object recognition memory: neurobiology, test procedure, and its modifications. Cogn Process. 2012, 13(2), 93110.
30. Orsetti M, Colella L, Dellarole A, Canonico PL, Ghi P. Modification of spatial recognition memory and object discrimination after chronic administration of haloperidol, amitriptyline, sodium valproate or olanzapine in normal and anhedonic rats. Int J Neuropsychopharmacol. 2007, 10(3), 345-57.

31. Saeed A, Shakir L, Khan MA, Ali A, Zaidi AA. Haloperidol Induced Parkinson's Disease Mice Model and Motor-Function Modulation with Pyridine-3-Carboxylic Acid. Biomed. Res. Ther. 2017, 4 (05), 1305.

32. Boulay D, Depoortere R, Oblin A, Sanger DJ, Schoemaker H, Perrault G. Haloperidol-Induced Catalepsy Is Absent in Dopamine D2, but Maintained in Dopamine D3 Receptor Knock-out Mice. Eur. J. Pharmacol. 2000, 391 (1-2), 63-73.

33. Adedeji HA, Ishola IO, Adeyemi OO. Novel Action of Metformin in the Prevention of Haloperidol-Induced Catalepsy in Mice: Potential in the Treatment of Parkinson's Disease? Prog. Neuropsychopharmacol. Biol. Psychiatry 2014, 48, 245-251.

34. Ramírez-Jarquín UN, Shahani N, Pryor W, Usiello A, Subramanian, S. The Mammalian Target of Rapamycin (MTOR) Kinase Mediates Haloperidol-Induced Cataleptic Behavior. Transl. Psychiatry 2020, 10 (1), 22.

35. Li W, Chaudhari K, Shetty R et al. Metformin Alters Locomotor and Cognitive Function and Brain Metabolism in Normoglycemic Mice. Aging Dis. 2019, 10 (5), 949.

36. Zemdegs $\mathrm{J}$, Martin $\mathrm{H}$, Pintana $\mathrm{H}$ et al. Metformin Promotes Anxiolytic and Antidepressant-Like Responses in Insulin-Resistant Mice by Decreasing Circulating Branched-Chain Amino Acids. J Neurosci. 2019, 39(30), 5935-5948.

37. Alfaras I, Mitchell SJ, Mora $\mathrm{H}$ et al. Health benefits of late-onset metformin treatment every other week in mice. NPJ Aging Mech Dis. $2017,3,16$. 